\title{
GIANT UTERINE LEIOMYOMA IN A YOUNG WOMAN AS AN ACCIDENTAL FINDING DUE TO AN ULTRASOUND FOR DIGESTIVE SYMPTOMS CAUSED BY ACUTE EXACERBATION OF CHRONIC GASTRITIS - A CASE REPORT
}

\author{
ALEXANDRU LASLO ${ }^{1}$ \\ 1 "Emil Palade" University of Medicine, Pharmacy, Science and Technology of Târgu-Mureş
}

\begin{abstract}
Keywords: giant uterine leiomyoma, hysterectomy, bulky tumour, necrosis

Abstract: Uterine leiomyoma is the most common benign tumour encountered in female genital pathology. It originates from the level of the smooth muscle tissue, and from the morphological point of view, the tumour is well delimited, being surrounded by a pseudocapsule. We report a case of a 46 year old woman who, following a CT scan performed for epigastric abdominal pain and nausea, showed a dense bulky tumour formation, with multiple hyperdense inclusions inside and well-defined areas of necrosis, with maximum axial diameters of 133/168/249 $\mathrm{mm}$ (LL / AP / CC), having as a starting point most likely the uterine upper pole. A total hysterectomy is performed, with bilateral anexectomy, and the histopathological examination describes a giant subserosal leiomyofibroma of the uterus with signs of hyaline degeneration, left hydrosalpinx with strong stasis of the left tube, follicular hemorrhagic cysts of the left ovary.
\end{abstract}

\section{INTRODUCTION}

Uterine leiomyoma is the most common benign tumour encountered in female genital pathology and its origins are from smooth muscle tissue.(1,2) Morphologically, the tumour is well delimited, being surrounded by a pseudocapsule formed by areolar tissue and compressed fibers. $(3,4)$ From the incidence point of view, the uterine leiomyoma was not described in the prepubertal period and it is found exceptionally in the case of female teenagers. $(5,6)$ In case of a woman at a reproductive age, the presence of asymptomatic leiomyoma is estimated at $40-50 \%$ of the females over 35 years old with a percentage that can reach up to $80 \%$ in the African-American population at the age of 50.(7) The incidence and severity of symptomatology are reduced after menopause in parallel with the disappearance of menstrual cycle and reducing circulating levels of sexual steroids. It is known that the black race has a three times higher risk of developing this pathology than the Caucasian people.(8)

It has been found in some studies the fact that one or more pregnancies exceeding twenty weeks of gestation decrease the risk of uterine leiomyomas and also smoking has a protective effect in the appearance of this pathology. $(8,9)$ Precocious menarche, at an age bellow 10 years old increases the risk of developing uterine leiomyomas, also as an increased consumption of red meat.(9) Abnormal uterine bleeding is the most common symptom and it can manifest as hypermenorrhea or menorrhagia which over time can lead to iron deficiency anemia. $(9,10)$ Most commonly, uterine leiomyomas with submucosal localization are accompanied by this symptomatology.(10)

In the case of a giant uterine leiomyoma, the pelvic painful symptoms may appear, due to the increased uterine volume and its irregular surface. Urinary symptoms may also be associated due to bladder compression, constipation due to rectal compression and many other symptoms depending on the organs affected by the tumour expansion process. $(10,11)$

The treatment of this pathology is preferable to be surgical, being accessible several options each adapted to the case.(11) Myomectomy is a conservative surgery indicated in cases where the patient wants to keep the reproductive system functional, procedure that involves the removal of one or more myomatous nodes with subsequent restoration of the uterine structure. $(11,12)$ As a disadvantage of this therapeutic approach is the fact that the source of leiomyomas is preserved so at 5 years postop $50-60 \%$ of these women will have new visible fibroids at ultrasound and about $10-25 \%$ of these will require surgical treatment. $(13,14,15)$ Hysterectomy is the most attractive option for women who have completed their family and whose fertility is no longer in question. This therapeutic approach helps not only on the symptoms given by the damage to the neighbouring organs trough compression but also on the danger in recurrence of the tumour formation.(12)

\section{CASE REPORT}

We report a case of a 46-year old woman known with chronic gastritis, pathology that was associating moderate digestive symptoms in spring and autumn, reason for which patient did not go to a doctor for an examination. Recently, the digestive symptoms such as abdominal pain mainly in the epigastric area and nausea have increased, reason for which the patient went to the family doctor for an examination. The family doctor decides to perform an abdominal ultrasound that shows a giant abdominal tumour mass with necrosis areas, the patient being guided to perform a CT scan.

Ct scan without oral or intravenous contrast substance in the context of the patient's allergic field reveals a voluminous

${ }^{1}$ Corresponding author: Alexandru Laslo, Str. Braşovului, Nr. 1, Ap. 227, Târgu-Mureş, România, E-mail: alexadru.laslo@gmail.com, Phone: +40747 278866

Article received on 12.05.2020 and accepted for publication on 10.08.2020 
dense tumour mass with multiple hypodense inclusions inside (necrosis areas) (figure no. 1) well delimited with maximum axial diameters of $14 \times 17 \times 25 \mathrm{~cm}$ (figure no. 2,3,4) having as starting point most likely the upper uterine pole without retroperitoneal lymphadenopathy, without ascites. The patient presents to the gynecology department for surgical treatment.

Total hysterectomy with bilateral annexectomy is performed, due to the large tumour volume and the fact that the patient does not want to maintain the fertility function. Postop, under treatment with fraxiparin, algocalmin and ketone, the evolution is favourable and allows the patient's discharge in general good health. One week after discharge, digestive symptoms reappear due to chronic gastritis, this explaining that the giant uterine leiomyoma did not lead to digestive symptoms; it practically caused no symptoms, which is our case particularity.

\section{Figure no. 1. CT scan that shows areas of necrosis}

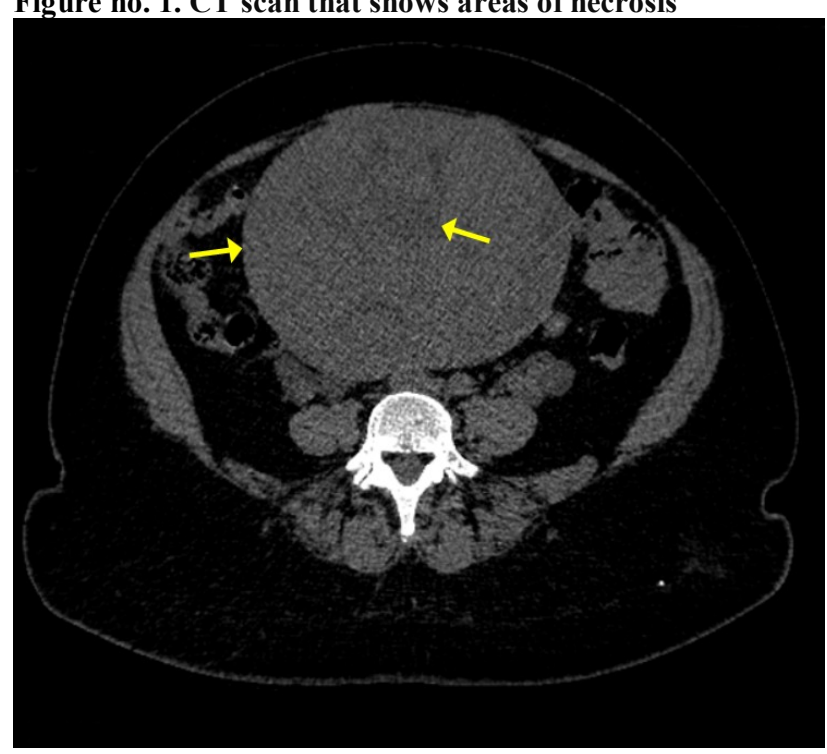

Figure no. 2. CT scan that shows the dimension of the tumour

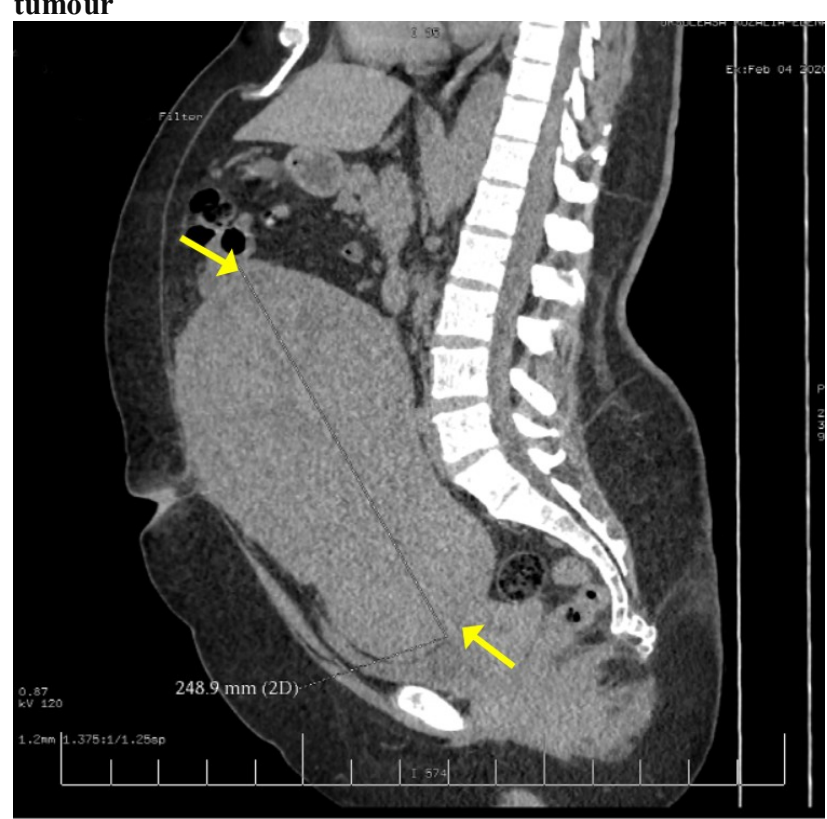

RESULTS

The macroscopic description in the histopathological examination show us: at the level of the uterine fundus, a giant tumour formation of $18 \times 15 \times 9 \mathrm{~cm}$ with a total weight of $2.45 \mathrm{~kg}$, deformed cervix $2 \times 2 \times 10 \mathrm{~cm}$ with an external dehiscent orifice, central cervical canal located with slightly irregular surface, deformed uterus $7 \times 13 \times 7 \mathrm{~cm}$ with a total weight of 381 grams, the slightly eccentric uterine cavity located with a macroscopic appearance in the slit, narrow, myometrium with a thickness of $0.5 \mathrm{~cm}$ is reduced around 3 tumours formations with the appearance of leiomyofibroma with dimensions $5 \times 5 \times 5 / 4 \times 4 \times 4$ $\mathrm{cm}$ and $1 \times 1 \times 1 \mathrm{~cm}$. At the level of uterine fundus, a giant tumour formation of $12 \times 11 \times 11 \mathrm{~cm}$ with a weight of $2.17 \mathrm{~kg}$ with characteristic appearance of uterine leiomyoma is identified. Left annex: $8 \times 1 \mathrm{~cm}$ tube with dilated lumen, paratubular an ovary of $3.5 \times 2 \times 2 \mathrm{~cm}$ with a weight of 20 grams, irregular surface, cystic structure with a hemorrhagic content.

Figure no. 3. CT scan that shows the dimension of the tumour

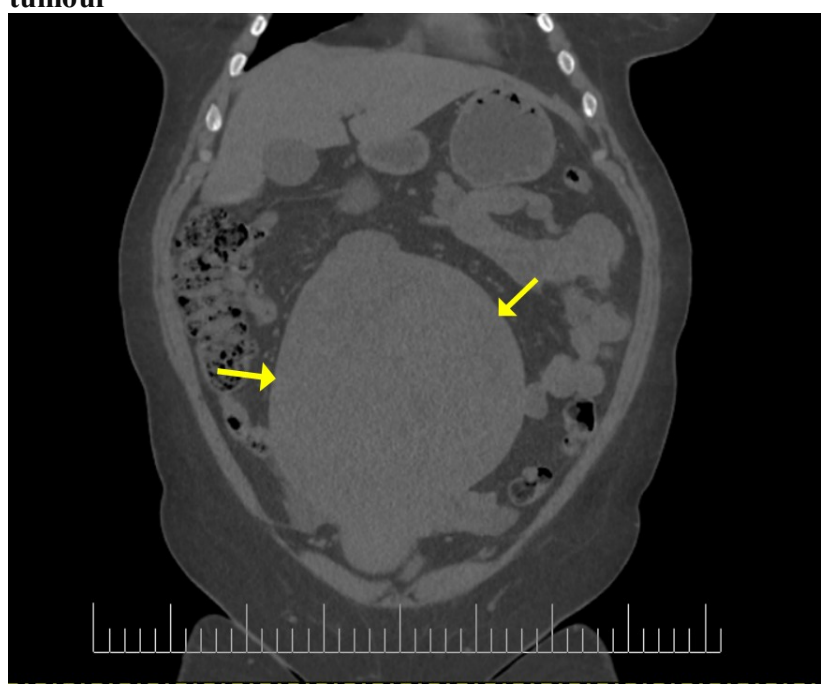

Figure no. 4. CT scan that shows topographic place extension

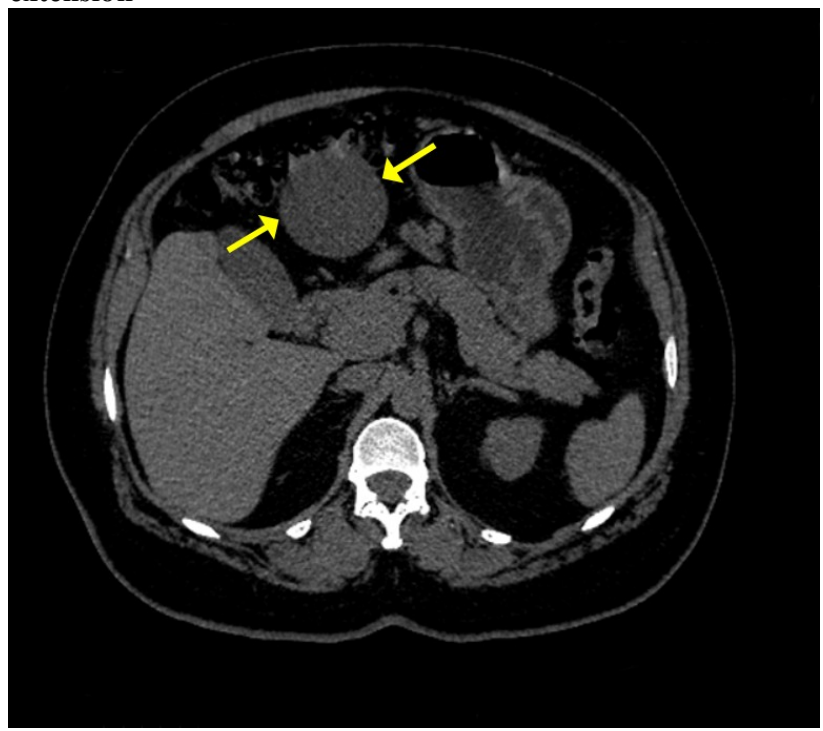

Right annex: $9 \times 15 \mathrm{~cm}$ tube with a sinuous trajectory, paratubular an ovary of $4 \times 3 \times 2 \mathrm{~cm}$ with a weight of 20 grams with a smooth, glossy surface, cystic structure with a hemorrhagic content.

The microscopic description of the histopathological examination shows us: cervix with rare retention cysts, endometrium corresponding to a weak estrogenic effect and 


\section{CLINICAL ASPECTS}

signs of its autolysis, intramural leiomyofibromatosis of the uterine body, no signs of secondary degeneration, giant subserosal leiomyoma of the uterine fundus with signs of hyaline degeneration, hemorrhagic follicular cyst of the left ovary, vascular stasis of the left and right tube, corpus albicans, corpus fibrosum and hemorrhagic follicular cyst of the right ovary.

\section{DISCUSSIONS}

Uterine leiomyomas are the most common diagnosis in the practice of gynaecological pathology. Often, these tumours can reach quite large sizes, but a leiomyoma as large as presents in this article is quite rare. In the speciality literature there is no well-defined criterion for classifying a leiomyoma as giant, but most authors who have published articles on giant uterine leiomyomas have described it as being more than $20 \mathrm{~cm}$ diameter.

The most common symptoms described by other authors were abnormal uterine bleeding, localized pain and abdominal distension.(16) None of these symptoms were found in the presented case which is a case particularity. After surgical removal of the uterus, the digestive symptoms most likely due to an acute exacerbation of chronic gastritis persists which is why we realized that the giant uterine leiomyoma does not affect neighboring organ systems as usually happens and as described by most authors in the speciality literature.

The lack of symptoms in giant uterine leiomyomas is due to the large volume of the abdominal cavity, slow growth rate of these tumours and distension of the abdominal wall. Usually, subserosal leiomyomas, as in our case are asymptomatic for a long time and sometimes reach large sizes without the patient noticing changes.(16)

Hydronephrosis, voiding problems and urinary tract infections can be detected, problems caused by the obstruction of urinary system due to compressive effect of a giant uterine leiomyoma. Novi JM et al. reported a case of a 46-year-old woman who presented acute urinary infection due to the obstruction of bladder outlet by uterine leiomyoma.(17) Also plethora can be a sign of uterine leiomyoma: Ozsaran AA et al. described a case of a giant subserosal uterine leiomyoma in which the patient presented plethora and abdominal mass.(18)

\section{CONCLUSIONS}

Giant uterine leiomyomas are a rare pathology in gynecological practice, but being often found accidentally and with atypical symptoms; it represents a great diagnostic challenge for clinicians. Histopathological examination is essential to establish the benign nature of the tumour and its differentiation from a malignant tumour such as leiomyosarcoma. Hysteretcomy is the treatment of choice for preventing tumour recurrence in such cases.

\section{REFERENCES}

1. Dragomir AD, Schroeder JC, Connolly A, et al. Potential risk factors associated with subtypes of uterine leiomyomata. Reprod Sci. 2010;17:1029.

2. Enkatachalam S, Bagratee JS, Moodley J. Medical management of uterine fibroids with medroxyprogesterone acetate (Depo Provera): a pilot study. J Obstet Gynaecol. 2004;24:798.

3. Parazzini F, Negri E, La Vecchia C, et al. Uterine myomas and smoking. Results from an Italian study. J Reprod Med. 1996;41:316.

4. Chiaffarino, F, Parazzini, F, La Vecchia, C, et al. Diet and uterine myomas. Obstet Gynecol. 1999;94:395.

5. Wise LA, Palmer JR, Harlow BL, et al. Risk of uterine leiomyomata in relation to tobacco, alcohol and caffeine consumption in the Black Women's Health Study. Hum Reprod. 2004;19:1746.

6. Donnez J, Jadoul P. What are the implications of myomas on fertility? A need for a debate?. Hum Reprod. 2002;17:1424

7. Marshall LM, Spiegelman D, Barbieri RL et al. Variation in the incidence of uterine leiomyoma among premenopausal women by age and race. Obstet Gynecol. 1997;90:967

8. Yoshida M, Koshiyama M, Fujii H, Konishi M. Erythrocytosis and a fibroid. Lancet. 1999;354:216.

9. Syrop CH, Nygaard IE, Davis WA. Natural history of uterine polyps and leiomyomata. Obstet Gynecol. 2002;100:3.

10. Viswanathan $\mathrm{M}$, Hartmann $\mathrm{K}$, McKoy $\mathrm{N}$, et al. Management of uterine fibroids: an update of the evidence. Evid Rep Technol Assess (Full Rep); 2007.

11. Minaguchi H, Wong JM, Snabes MC. Clinical use of nafarelin in the treatment of leiomyomas. A review of the literature. J Reprod Med. 2000;45:481.

12. Eisinger SH, Meldrum S, Fiscella $\mathrm{K}$, et al. Low-dose mifepristone for uterine leiomyomata. Obstet Gynecol. 2003;101:243

13. Hanafi M. Recurrence after removal of solitary and multiple myomas. Obstet Gynecol. 2005; 105:877.

14. Hald K, Langebrekke A, Klow NE, et al. Laparoscopic occlusion of uterine vessels for the treatment of symptomatic fibroids: Initial experience and comparison to uterine artery embolization. Am J Obstet Gynecol. 2004:190:37

15. DeCherney AH, Nathan L. Current obstetric \& gynecologic diagnosis \& treatment. 9th ed. New York (NY): Lange Medical/McGraw-Hill; 2003. p. 708-715.

16. Vladimír B, Peter K, Robert F. Giant Uterine Leiomyoma in a Young Woman as an Incidental Finding After a Car Accident: A Case Report. Acta Medica (Hradec Králové) 2018;61(1):29-32.

17. Novi JM, Shaunik A, Mulvihill BH, Morgan MA. Acute urinary retention caused by a uterine leiomyoma: a case report, J Reprod Med. 2004;49:131-132.

18. Ozsaran AA, Itil IM, Terek C, Kazandi M, Dikmen Y. Giant myoma and erythrocytosis syndrome, Aust N Z J Obstet Gynaecol. 1999;39:384-386. 